\title{
Chronic Pelvic Pain: An Overview
}

\author{
Jai Kishan Goel, Siddharth Goel
}

\section{ABSTRACT}

Chronic pelvic pain (CPP) is not a disease but a complex multidimensional syndrome. Although any one disorder may be the cause of CPP, pain can also be the end result of several medical conditions, with each contributing to the generation of pain and requiring management.

Women with chronic pelvic pain are often disappointed with the quality of their medical consultations and want their clinician, a personalized care, empathetic and take their symptoms seriously, provide an explanation as to the cause of their pain syndrome and be reassuring. Gastrointestinal, urological, gynecological, psychological, and musculoskeletal sources should be considered in evaluating women with chronic pelvic pain. A complete history, physical examination and counseling can take 45 to 90 minutes to women with CPP. Laboratory testing is of limited value in evaluating women with CPP and, however, pelvic ultrasound is highly sensitive for identifying pelvic masses and determining the origin of the mass. Diagnostic laparoscopy is recommended, if $\mathrm{Gl}, \mathrm{GU}$ and myofascial and neuropathic causes are ruled out or treated and the results of the psychological evaluation are negative. However in $30 \%$ of the cases, no cause is ever determined and it presents a therapeutic challenge to the clinician.

Success in treating women with chronic pelvic pain is greatly facilitated by earning their trust and confidence. To decide on the best therapeutic plan for an individual patient, the physician and patient should have a thorough discussion of her preferences and values regarding testing, medical versus surgical treatment and childbearing plans. For many patients, the optimal approach involves a combination of treatments.

Keywords: Chronic, Pelvic pain, Evaluation, Multimodal therapy.

How to cite this article: Goel JK, Goel S. Chronic Pelvic Pain: An Overview. J South Asian Feder Obst Gynae 2013;5(3): 147-153.

\section{Source of support: Nil}

Conflict of interest: None declared

\section{INTRODUCTION}

Chronic pelvic pain is defined as pelvic pain that persists in the same location for greater than 6-month duration, causing functional disability or requiring treatment. ${ }^{1}$ Taking a detailed medical history, the nature, frequency and site of the pain and its relationship to precipitating factors, menstrual and sexual history are essential for making a diagnosis. Discrete inquiry about previous sexual trauma may be appropriate. Chronic pelvic pain in urological and gynecological practice is often complex and difficult to treat. In $30 \%$ of the cases, no cause is ever determined and this presents a therapeutic challenge and a source of frustration for both the patient and clinician. ${ }^{2}$

The prevalence of CPP ranges from 4 to $6 \%$, but only about one-third of women with chronic pelvic pain (CPP) seek medical care. ${ }^{3}$

\section{Etiology}

The major sources that contribute to pelvic pain are as follows:

- Gynecological

- Mental health issues

- Urological

- Gastrointestinal

- Musculoskeletal

- Neurological disorders

Conditions associated with chronic pelvic pain in women are shown in Table $1 .^{1}$

Some important gynecological causes are discussed below:

i. Endometriosis: It is the most common diagnosis made at the time of gynecological laparoscopy for the evaluation of CPP. Overall, about one-third of women who undergo laparoscopy because of CPP are diagnosed with endometriosis; however, in practices specializing in the treatment of endometriosis, $70 \%$ or more of patients with CPP are given this diagnosis.

Table 1: Conditions associated with chronic pelvic pain in women $^{1}$

\section{Gynecologic}

Endometriosis

Chronic pelvic inflammatory disease

Pelvic adhesions

Pelvic congestion (pelvic varicosities)

Adenomyosis

Ovarian remnant syndrome

Residual ovary syndrome

Leiomyoma

Endosalpingiosis

Neoplasia

Fallopian tubal prolapsed (posthysterectomy)

Tuberculous salpingitis

Benign cystic mesothelioma

Postoperative peritoneal cysts

Mental health issues

Somatization

Substance abuse

Physical and sexual abuse

Depression

Sleep disorders

Urinary tract

Interstitial cystitis/painful bladder syndrome

Recurrent urinary tract infection

Urethral diverticulum

Chronic urethral syndrome

Neoplasia

Radiation cystitis

Gastrointestinal tract

Irritable bowel syndrome

Inflammatory bowel disease and other causes of colitis

Diverticular colitis

Chronic intermittent bowel obstruction

Neoplasia

Chronic constipation

Celiac disease (sprue) 


\section{Contd...}

\section{Musculoskeletal}

Pelvic floor myalgia

Myofascial pain (trigger points)

Coccygodynia

Piriformis syndrome

Hernia

Abnormal posture

Fibromyalgia

Peripartum pelvic pain syndromes

\section{Neurologic disorders}

Neuralgia, especially of the iliohypogastric, illioinguinal, genitofemoral or pudendal nerves

Herniated nucleus pulposus

Neoplasia

Neuropathic pain

Abdominal epilepsies

Abdominal migraine

ii. Pelvic inflammatory disease: As many as $30 \%$ of women with pelvic inflammatory disease (PID) subsequently develop CPP. ${ }^{4}$ Therefore, PID is a common cause of CPP in settings with a high prevalence of sexually transmitted disease.

iii. Adhesions: The relationship between CPP and the presence of adhesions is poorly defined. There is some evidence that dense adhesions that limit organ mobility cause visceral pain, ${ }^{5}$ and evidence from conscious laparoscopic pain mapping that adhesions may account for pelvic pain in some patients

iv. Pelvic congestion syndrome: Pelvic congestion syndrome is a controversial entity. It refers to a condition in which characteristic symptoms of shifting location of pain, deep dyspareunia, postcoital pain and exacerbation of pain after prolonged standing are associated with radiological findings of pelvic varicosities (dilated uterine and ovarian veins) that display reduced blood flow. ${ }^{6}$

v. Adenomyosis: Abnormal uterine bleeding and dysmenorrhea are the major symptoms of adenomyosis. Pain may be due to bleeding and swelling of endometrial islands confined by myometrium. Symptoms typically develop between the ages of 40 and 50 years.

vi. Ovarian cancer: Ovarian cancer is not truly a 'silent killer'. Most affected women have one or more nonspecific symptoms, such as lower abdominal pain/ discomfort/pressure/bloating, increased abdominal size, constipation, lack of appetite/nausea/indigestion, irregular menstrual cycles/abnormal vaginal bleeding, low back pain, fatigue, urinary frequency or dyspareunia.

vii. Ovarian remnant and residual ovary syndrome: The ovarian remnant syndrome (ORS) occurs in patients who have undergone bilateral oophorectomy and, subsequently, present with symptoms related to ovulatory function from ovarian tissue inadvertently left behind. It should be distinguished from the residual ovary syndrome (ROS), in which the ovary was intentionally preserved and subsequently developed pathology. The typical patient presents with cyclic pelvic pain and a mass, although the pain may be persistent with acute flare-ups. Occasionally, an asymptomatic mass is detected on pelvic or sonographic examination. Ureteral obstruction may occur. viii. Leiomyoma: Uterine leiomyomas may cause pressure symptoms. Acute pain occurs with degeneration, torsion or expulsion through the cervix. Chronic pain is uncommon.

ix. Dysmenorrhea: Dysmenorrhea commonly occurs in women with CPP. Secondary dysmenorrhea suggests the development of a pathological process and it is essential to exclude endometriosis, adenomyosis ${ }^{7}$ and pelvic infection.

x. Others: Caronic pelvic pain is often associated with vulvar pain and dyspareunia. It can also be due to postsurgical neuropathy, such as nerve entrapment. Abdominal cutaneous nerve injury or entrapment may occur spontaneously or within weeks to years after transverse suprapubic skin or laparoscopy incisions. Femoral nerve injury, one of the most commonly injured nerves in gynecologic laparotomy, can result when deep lateral retractor blades compress the nerve between the blade and the lateral pelvic side wall. ${ }^{8}$

\section{Evaluation of CPP}

On the first visit, a thorough pain history should be performed, taking into consideration the nature of each pain symptom. Pain history of mnemonic is given in Table 2 . $^{1}$

Diagram of woman is abdomen back and genital area should be used to help the patient to define the location of pain. A patient should be questioned about symptoms specific to the types of pathology.

1. Genital (abnormal vaginal bleeding, abnormal vaginal discharge, dysmenorrhea, dyspareunia, subfertility, sexual functioning).

\begin{tabular}{|c|c|}
\hline Old carts & Pain history \\
\hline Onset & $\begin{array}{l}\text { When and how did pain start? Did } \\
\text { it change over time? }\end{array}$ \\
\hline Location & $\begin{array}{l}\text { Localize specifically. Can you put a } \\
\text { finger on it? }\end{array}$ \\
\hline Duration & How long does it last? \\
\hline Characteristic & $\begin{array}{l}\text { Cramping, aching, stabbing, } \\
\text { burning. 'Like lightening,' tingling, } \\
\text { itching }\end{array}$ \\
\hline $\begin{array}{l}\text { Alleviating/aggravating } \\
\text { factors }\end{array}$ & $\begin{array}{l}\text { What makes it better (medication, } \\
\text { stress reduction, heat/ice, position } \\
\text { change) or worse (specific activity, } \\
\text { stress, menstrual cycle)? }\end{array}$ \\
\hline Associated symptoms & $\begin{array}{l}\text { Gynecologic (dyspareunia, } \\
\text { dysmenorrhea abnormal bleeding, } \\
\text { discharge, infertility), GI } \\
\text { (constipation, diarrhea, bloating, } \\
\text { gas, rectal bleeding), GU } \\
\text { (frequency, dysuria, urgency, } \\
\text { incontinence), neurologic (specific } \\
\text { nerve distribution) }\end{array}$ \\
\hline Radiation & $\begin{array}{l}\text { Does it move to other areas } \\
\text { (dermatomal)? }\end{array}$ \\
\hline Temporal & $\begin{array}{l}\text { What time of day (elation to } \\
\text { menstrual cycle and activities of } \\
\text { daily living)? }\end{array}$ \\
\hline Severity & Scale of $0-10$ \\
\hline
\end{tabular}


2. Enterocoelic (constipation, diarrhea, flatulence, hematochezia and relationship of pain to times of altered bowel function or form and pain relief with bowel movements).

3. Musculoskeletal/neuropathic (physical trauma - surgical or injury, exacerbation with exercise or postural changes, weakness, numbness, lancinating pain).

4. Urologic (urgency, frequency, nocturia, hesitancy, dysuria, incontinence).

5. Psychological (previous diagnoses, hospitalizations and medications, current depression, anxiety, panic, including suicidal ideation, past and current emotional, physical or sexual trauma).

Use of the International Pelvic Pain Society's form facilitates obtaining the history and performing the physical examination. Asking the patient to complete a pain map can also be helpful for localizing pain.

The goal of the evaluation is to identify tender areas, correlate these areas with the patient's pain map and determine whether the pain produced on examination represents her CPP.

Laboratory testing is of limited value in evaluating women with CPP. A complete blood count with differential count, erythrocyte sedimentation rate, urinalysis, testing for chlamydia and gonorrhea infection and a pregnancy test to exclude pregnancy.

Pelvic ultrasound is highly sensitive for identifying pelvic masses and determining the origin of the mass (ovary, uterus, fallopian tube).

Magnetic resonance imaging (MRI) is obtained in some cases to define better, an abnormality suspected by sonography ${ }^{9}$ and for diagnosis of adenomyosis.

Diagnostic laparoscopy is recommended, if GI, GU and myofascial and neuropathic causes are ruled out or treated and the results of the psychological evaluation are negative.

\section{Treatment}

Success in treating women with CPP is greatly facilitated by earning their trust and confidence.

To decide on the best therapeutic plan for an individual patient, the physician and patient should have a thorough discussion of her preferences and values regarding testing, medical versus surgical treatment and childbearing plans. For many patients, the optimal approach involves a combination of treatments.

The following therapeutic plans/modalities are available:

- Empiric trial of therapy based on diagnostic probabilities.

- Intensive diagnostic evaluation followed by targeted therapy.

- Nonspecific analgesia.

Empiric trial of therapy based on diagnostic probabilities: In these approach, sequential drug treatments for disorders that are the most likely causes of the patient's CPP are prescribed. Endometriosis is the most common gynecological cause of CPP. If the diagnostic impression is most consistent with endometriosis after a complete initial evaluation, then most experts consider empiric medical therapy without surgical confirmation a safe and effective approach.

First line agents employed sequentially include the following: ${ }^{10}$

- Nonsteroidal anti-inflammatory drugs (NSAIDs) prescribed at maximal doses (e.g. ibuprofen $800 \mathrm{mg}$ orally every 8 hours).

- Oral contraceptive pills (OCs) prescribed as monthly cycles for two to three months. ${ }^{11}$

- OCs prescribed continuously, i.e. without a break to allow menstrual flow. This regimen has been effective in women who failed cyclic therapy. ${ }^{12}$

If the combination of NSAIDs and continuous OCs is unsuccessful after 2 to 3 months, then second line agents should be tried.

Table 3: Summary of recommendations for treatment of chronic pelvic pain from the American College of Obstetricians and Gynecologists $^{1}$

Recommendations based on consistent scientific evidence (level A)

\begin{tabular}{|c|c|}
\hline Intervention & Indication \\
\hline $\begin{array}{l}\text { Combined oral } \\
\text { contraceptive pills }\end{array}$ & Primary dysmenorrhea \\
\hline $\mathrm{GnRH}$ agonists & $\begin{array}{l}\text { Endometriosis, irritable bowel } \\
\text { syndrome (may be given } \\
\text { empirically in women with } \\
\text { symptoms consistent with } \\
\text { endometriosis) }\end{array}$ \\
\hline $\begin{array}{l}\text { Nonsteroidal anti- } \\
\text { inflammatory drugs }\end{array}$ & Dysmenorrhea, moderate pain \\
\hline $\begin{array}{l}\text { Progestins (daily, high } \\
\text { dose) }\end{array}$ & $\begin{array}{l}\text { Endometriosis, pelvic congestion } \\
\text { syndrome }\end{array}$ \\
\hline $\begin{array}{l}\text { Laparoscopic ablation/ } \\
\text { resection of endometriosis }\end{array}$ & Stage I - III endometriosis \\
\hline Presacral neurectomy & Centrally I menorrhea \\
\hline Uterine nerve ablation & Not indicated \\
\hline Adjunctive psychotherapy & CPP \\
\hline
\end{tabular}

Recommendations based on limited or inconsistent scientific evidence (level B)

\begin{tabular}{|c|c|}
\hline Intervention & Indication \\
\hline GnRH agonists & CPP other than endometriosis \\
\hline Adhesiolysis & $\begin{array}{l}\text { CPP attributed to dense bowel } \\
\text { adhesions }\end{array}$ \\
\hline Hysterectomy & Reproductive tract symptoms \\
\hline Sacral nerve stimulation & CPP \\
\hline Physical therapy & CPP \\
\hline $\begin{array}{l}\text { Nutritional supplementation } \\
\text { with vitamin B1 or } \\
\text { magnesium } \\
\text { Injection of trigger points }\end{array}$ & Dysmenorrhea \\
\hline $\begin{array}{l}\text { Application of magnets to } \\
\text { trigger points }\end{array}$ & CPP \\
\hline $\begin{array}{l}\text { Acupuncture, acupressor, } \\
\text { transcutaneous nerve } \\
\text { stimulation }\end{array}$ & Primary dysmenorrhea \\
\hline \multicolumn{2}{|c|}{$\begin{array}{l}\text { Recommendation based on consensus and expert opinion } \\
\text { (level C) }\end{array}$} \\
\hline Intervention & Indication \\
\hline Antidepressants & CPP \\
\hline Opioids & CPP \\
\hline
\end{tabular}


- Continuous progestin treatments that have shown efficacy in randomized clinical trials include. ${ }^{13}$

- Medroxyprogesterone acetate (initially at $10 \mathrm{mg}$ daily and may increase up to $50 \mathrm{mg}$ orally daily depending on tolerance).

- Norethindrone acetate (initially at $2.5 \mathrm{mg}$ orally daily and may increase to $30 \mathrm{mg}$ orally daily).

- Subcutaneous depo-medroxyprogesterone acetate, $104 \mathrm{mg}$ every 3 months.

- A levonorgestrel-releasing intrauterine system-in a randomized clinical trial, this device reduced pelvic pain in women with endometriosis with similar success as a GnRH agonist. ${ }^{13}$

- GnRH agonist (e.g. leuprolide acetate $3.75 \mathrm{mg}$ intramuscularly every 4 weeks $^{14}$ or $11.25 \mathrm{mg}$ intramuscularly every 12 weeks).

- Danazol 200 to $400 \mathrm{mg} /$ day total divided into two doses. The dose may be increased to a total dose of $800 \mathrm{mg} /$ day to achieve amenorrhea and rapid response to painful symptoms.

Surgical evaluation and treatment via laparoscopy is the next option, if empiric medical interventions are not successful. Surgery can be performed instead of empiric medical therapy, if the patient prefers and should be the initial approach, if a pelvic mass is detected (to exclude neoplasia).

Targeted treatments: Targeted therapy is appropriate if a specific diagnosis has been made.

Therapeutic options are briefly summarized below:

Chronic pelvic inflammatory disease: Women with a prior history of pelvic inflammatory disease are at significant risk for subsequent CPP. If ongoing or subclinical infection is suspected, antibiotic treatment should be initiated. However, there is no high-quality evidence of efficacy of empiric antibiotic treatment. Standard broad-spectrum antibiotics targeting Gram positive and negative organisms are normally recommended.

Similarly, there are no high quality clinical trials of surgical treatment for CPP after pelvic inflammatory disease. In the absence of good data, such surgical approaches must be weighed carefully against the short- and long-term potential risks.

Pelvic congestion syndrome: Although this diagnosis remains controversial, there is evidence that some women with pelvic varicosities and venous stasis, as demonstrated by pelvic venography, have associated CPP. ${ }^{15}$

Two randomized clinical trials suggest that medical treatment with gonadotropin-releasing hormone agonists or synthetic progestins effectively decrease pain associated with pelvic congestion: ${ }^{16}$

Other treatments for pelvic congestion syndrome which have not been subjected to randomized clinical trials include the following:

- Hysterectomy and bilateral salpingo-oophorectomy ${ }^{17}$

- Embolization of the ovarian veins with or without the internal iliac veins ${ }^{18}$

- Sclerotherapy $^{19}$

- Surgical ligation of the ovarian veins. ${ }^{20}$
Efficacy of these treatments is supported only by observational data and case reports. For embolization, such studies have reported technical success rates of 89 to $97 \%$ and clinical success rates of 74 to $89 \%$ over a follow-up period of up to 5 years. ${ }^{21}$

Adenomyosis: Hysterectomy is the treatment of choice for women with significant symptoms from adenomyosis. There is anecdotal evidence that gonadotropin-releasing hormone agonist treatment or insertion of a levonorgestrel-releasing intrauterine device may be effective treatments, but clinical studies are needed.

Ovarian remnant syndrome, retained ovary syndrome: Initial medical treatment with high-dose progestins or oral contraceptives usually provides good results. Pain relief can also be achieved with GnRH agonists but long-term therapy is impractical. Removal of the remnant ovarian tissue is necessary for treatment, and the corrective surgery tends to be arduous. Clomiphene citrate can be used 7 to 10 days before surgery to induce folliculogenesis, allowing ovarian tissue to be more easily detected.

Leiomyoma: Removal or reduction in size of leiomyomas can relieve chronic pressure symptoms. Chronic pelvic pain is an uncommon manifestation of leiomyomas, thus, treatment of these tumors for relief of chronic pain is not usually indicated. Treatment recommendations for management of uterine leiomyomas are primarily based upon outcomes described in nonrandomized, and often uncontrolled, reports. Choice of treatment modality is based upon the size and location of the leiomyomas, types of leiomyoma-related symptoms, age of the patient and patient preference (desire for uterine conservation versus definitive therapy).

Vulvar pain, dyspareunia: Although therapeutic options remain limited and require a multidisciplinary pain management approach with psychological and physiotherapy input. These can be treated effectively with physiotherapy, streatching exercises and even botulinum toxin, though in the latter case the evidence is variable.

Dysmenorrhea: Reassurance and an explanation of the cause of dysmenorrhea are usually helpful, together with the use of simple analgesics, followed by nonsteroidal antiinflammatory drugs (NSAIDs), ${ }^{22}$ which are particularly helpful if they are started before the onset of each menstrual cycle. NSAIDs are effective in dysmenorrhea, probably because of their effects on prostaglandin synthetase.

Suppression of ovulation using oral contraceptive tablets (either combined or progesterone only) or the use of a levonorgestrel intrauterine device reduces dysmenorrhea dramatically in most cases and may be used as a therapeutic test.

Adhesions: The best available evidence showed adhesiolysis is not effective for relieving chronic pain in most women with CPP or chronic abdominal pain, although observational studies have suggested efficacy. This conclusion was supported by randomized controlled trials. ${ }^{23}$

Irritable bowel syndrome: Modifications in diet, behavioral changes, medications and psychotherapy are utilized alone or in combination, based on the severity of symptoms. 
Interstitial cystitis/painful bladder syndrome: There is no definitive cure for IC/BPS, remission can be achieved by multidisciplinary therapy. First line treatment are preliminary behavioral modification, such as bladder training and stress management, cognitive-behavioral therapy, dietary modifications or restriction of acidic, spicy and fermented foods and pelvic floor muscle physical therapy. Urinary alkalinization can be useful. TCAs have been efficacious. Oral pentosan polysulfate sodium (PPS) is the only US food and Drug Administration (FDA) approved oral therapy for IC and is modestly beneficial.

Intravesical therapy with a mixture of lidocaine, bicarbonate and heparin instilled three times a week for 3 weeks. Dimethyl sulfoxide (DMSO) 50\% used intravesical showed significant symptomatic relief. Sacral neuromodulation is an invasive, yet promising, technique under investigation for treatment of refractory IC. ${ }^{24}$

Myofascial pain syndrome (trigger point pain): Although abdominal and pelvic myofascial pain and focal tender points consistent with trigger points are often noted in patients with CPP.

The approaches that we think are most effective for myofascial pain include the following:

- Physical therapy: Icing, moist heat, stretching, massage, ultrasound, transcutaneous electrical stimulation, galvanic muscle stimulation and biofeedback are the mainstay of treatment. Sustained pressure with adequate force to a trigger point for a specified period can inactivate the irritable nerve. $^{25}$

- Trigger point injections: The dose is 1 to $5 \mathrm{ml}$ of a local anesthetic (e.g. $1 \%$ lidocaine or $0.25 \%$ bupivacaine) at each trigger point, with or without glucocorticoid (e.g. triamcinolone 10 to $40 \mathrm{mg}$ per trigger point). ${ }^{25}$

- Local anesthetic patches: Efficacy of a 5\% lidocaine patch at the area of trigger point pain has been supported by at least one placebo-controlled randomized trial. ${ }^{26}$

- Acupuncture: Direct needle insertion at the site of myofascial pain may be of some benefit.

The authors concluded that most of the therapeutic response after acupuncture is due to placebo effects.

Mental health issues: Mental health issues should be addressed and managed, as appropriate.

Depression is common in women with CPP and warrants treatment. However, data regarding the efficacy of antidepressants in the treatment of pain in CPP are limited.

Somatization disorder: Cognitive behavioral therapy (CBT) is the best established treatment for a variety of somatoform disorders, including somatization disorder. ${ }^{27}$

The use of antidepressants is preliminary but does not yet show conclusive evidence. Electroconvulsive shock therapy has been used in treating somatization disorders among the elderly; however, the results are still debatable with some concerns around the side effects of using ECT.

Post-traumatic stress disorders (PTSD): Psychobiological treatments target biological changes that occur after a traumatic event. They also attempt to chemically alter learning or memory formation. Psychobiological treatments are found to be of success, especially with cortisol. The psychotherapy programs with the strongest demonstrated efficacy include cognitive behavioral programs, variants of exposure therapy, stress inoculation training (SIT), variants of cognitive therapy (CT), eye movement desensitization and reprocessing (EMDR), and many combinations of these procedures. ${ }^{28}$ A variety of medications have shown adjunctive benefit in reducing PTSD symptoms, but there is no clear drug treatment for PTSD.

Substance use disorder: Treatment usually involve planning for specific ways to avoid the addictive stimulus, and therapeutic interventions intended to help a client learn healthier ways to find satisfaction. Clinical leaders in recent years have attempted to tailor intervention approaches to specific influences that affect addictive behavior, using therapeutic interviews in an effort to disorder factors that led a person to embrace unhealthy, addictive sources of pleasure or relief from pain.

Nonspecific treatments: For patients who fail or have an inadequate response to targeted treatments or when no specific diagnosis can be made, nonspecific treatment may be initiated, i.e. directed to pain relief without regard to etiology. Such therapies may be pharmacological, surgical, psychological or neurological (nerve blocks or neurodestructive procedures).

Heat therapy: Heat can be useful, especially for musculoskeletal pain. Heat acts as an analgesic in part by increasing blood flow and decreasing joint stiffness. It also may induce a state of mental relaxation.

Drugs: Some patients with CPP have features of neuropathic pain. Neuropathic pain can be treated with lowdose amitriptyline, nortriptyline, gabapentin or narcotics. ${ }^{29}$

Hysterectomy: There are no randomized controlled trials evaluating the efficacy of hysterectomy in the treatment of CPP. Both prospective and retrospective cohort studies suggest that hysterectomy significantly relieves or eliminates CPP in 78 to $96 \%$ of women. ${ }^{30}$

Hysterectomy is not a good choice for the management of CPP in women who have not completed their family.

Nerve transection procedures: There are few high quality clinical trials examining the efficacy of nerve transection procedures for the treatment of CPP. Laparoscopic uterosacral nerve ablation (LUNA) and presacral neurectomy (PSN) are the procedures studied most intensively. Limited data suggest that LUNA is not a useful approach to the treatment of CPP, and that PSN may be modestly effective, but the success of the procedure may depend, in part, on the experience of the surgeon.

Lapacosopic presacral neurectomy (LPSN) appeared to be more effective than LUNA, although data were limited to one small randomized trial. In this trial, patients undergoing LPSN were significantly more likely to have relief of primary dysmenorrhea at 12 months than those who underwent LUNA.

Observational series have shown that success rates declined rapidly over time (years), possibly due to regrowth of nerves or pain signals being transferred via alternative routes. ${ }^{31}$

In summary, there is evidence that LUNA is not effective in the treatment of CPP, and more evidence is needed before LPSN can be recommended for treatment of CPP. 
Sacral nerve root neuromodulation: In general, neuromodulation for CPP has not been well-studied. Sacral nerve root neuromodulation for bladder-related symptoms and pain is the best studied technique, but all trials are observational.

There is some information on laparoscopic intraabdominal placement of a neuroprosthesis for CPP, but this work is still early in development and has not been widely used. ${ }^{32}$

Multidisciplinary approach: Although there is an opinion that treatment in a multispecialty pain practice prior to surgery is beneficial, this has not been as well studied in CPP as in other types of chronic pain. Pain services can develop a multimodal treatment plan that includes many of the treatments discussed above (e.g. drugs, trigger point injections) as well as:

- Psychological counseling

- Acupuncture

- Behavioral and relaxation feedback therapies

- Transcutaneous electrical nerve stimulation

- Implantable nerve stimulation devices.

Recommendations as opined by American College of Obstetricians and Gynecologists are shown in Table 3. ${ }^{1}$

\section{CONCLUSION}

The gastrointestinal and urinary tracts are the organ systems most often affected by disorders causing chronic pelvic pain which is quite annoying to the patient and equally annoying to the physician. Endometriosis is the most common gynecological cause of CPP. The goal in evaluating the chronic pelvic pain is to identify tender areas, correlate these areas with the patient's pain map. The use of laparoscopic in women with CPP should be individualized. Choosing the best treatment plan for chronic pelvic pain is based upon shared decision making by the clinician and the patient. A combination of the treatments may be the optimal approach. Multimodal therapy (medical, surgical and behavioral/mental health therapy along with pain consultation) is more effective than treatments that emphasize a single approach.

\section{REFERENCES}

1. ACOG Committee on Practice Bulletins. ACOG Practice Bulletin No 51. Chronic pelvic pain. Obstet Gynecol 2004;103:589-605.

2. Newham AP, Van Der Spuy ZM, Nugent F. Laparoscopic findings in women with chronic pelvic pain. S Afr Med J 1996 Sep;86(9 Suppl):1200-1203.

3. Zondervan KT, Yudkin PL, Vessey MP, et al. Prevalence and incidence of chronic pelvic pain in primary care: evidence from a national general practice database. Br J Obstet Gynaecol 1999; 106:1149.

4. Ness RB, Soper DE, Holley RL, et al. Effectiveness of inpatient and outpatient treatment strategies for women with pelvic inflammatory disease: Results from the Pelvic Inflammatory Disease Evaluation and Clinical Health (PEACH) Randomized Trial. Am J Obstet Gynecol 2002;186:929.

5. Practice Committee of the American Society for Reproductive Medicine, Society of Reproductive Surgeons. Pathogenesis, consequences and control of peritoneal adhesions in gynecologic surgery. Fertil Steril 2007;88:21.
6. Beard RW, Reginald PW, Wadsworth J. Clinical features of women with chronic lower abdominal pain and pelvic congestion. Br J Obstet Gynaecol 1988;95:153.

7. Wassong C, Shah B, Kanayama M, et al. Radiologic findings of pelvic venous congestion in an adolescent girl with angiographic confirmation and interventional treatment. Pediatr Radiol 2011 Sep;13.

8. Fardin F, Benettello P, Negrin P. Iatrogenic femoral neuropathy: Considerations on its prognosis. Electromyogr Clin Neurophysiol 1980;20:153-155.

9. Cody RF JR, Ascher SM. Diagnostic value of radiological tests in chronic pelvic pain. Baillieres Best Pract Res Clin Obstet Gynaecol 2000;14:433.

10. Kennedy S, Bergqvist A, Chapron C, et al. ESHRE guideline for the diagnosis and treatment of endometriosis. Hum Reprod 2005;20:2698.

11. Vercellini P, Trespidi L, Colombo A, et al. A gonadotropinreleasing hormone agonist versus a low-dose oral contraceptive for pelvic pain associated with endometriosis. Fertil Steril 1993; 60:75.

12. Vercellini P, Frontino G, De Giorgi O, et al. Continuous use of an oral contraceptive for endometriosis-associated recurrent dysmenorrhea that does not respond to a cyclic pill regimen. Fertil Steril 2003;80:560.

13. Petta CA, Ferriani RA, Abrao MS, et al. Randomized clinical trial of a levonorgestrel-releasing intrauterine system and a depot GnRH analogue for the treatment of chronic pelvic pain in women with endometriosis. Hum Reprod 2005;20:1993.

14. Ling FW. Randomized controlled trial of depot leuprolide in patients with chronic pelvic pain and clinically suspected endometriosis. Pelvic Pain Study Group. Obstet Gynecol 1999; 93:51.

15. Beard RW, Highman JH, Pearce S, Reginald PW. Diagnosis of pelvic varicosities in women with chronic pelvic pain. Lancet 1984;2:946.

16. Farquhar CM, Rogers V, Franks S, et al. A randomized controlled trial of medroxyprogesterone acetate and psychotherapy for the treatment of pelvic congestion. Br J Obstet Gynaecol 1989;96:1153.

17. Beard RW, Kennedy RG, Gangar KF, et al. Bilateral oophorectomy and hysterectomy in the treatment of intractable pelvic pain associated with pelvic congestion. Br J Obstet Gynaecol 1991 Oct;98(10):988-992.

18. Kim HS, Malhotra AD, Rowe PC, et al. Embolotherapy for pelvic congestion syndrome: long-term results. J Vasc Interv Radiol 2006;17:289.

19. Ghosh A, Shafie-Pour H, Ayers KJ. Laparoscopic sclerotherapy in a case of pelvic congestion syndrome. BJOG 2006;113:610.

20. Gargiulo T, Mais V, Brokaj L, et al. Bilateral laparoscopic transperitoneal ligation of ovarian veins for treatment of pelvic congestion syndrome. J Am Assoc Gynecol Laparosc 2003; 10:501.

21. Chung MH, Huh CY. Comparison of treatments for pelvic congestion syndrome. Tohoku J Exp Med 2003;201:131.

22. Allen C, Hopewell S, Prentice A, et al. Nonsteroidal antiinflammatory drugs for pain in women with endometriosis. Cochrane Database Syst Rev. 2009 Apr 15;(2):CD004753.

23. Peters AA, Trimbos-Kemper GC, Admiraal C, et al. A randomized clinical trial on the benefit of adhesiolysis in patients with intraperitoneal adhesions and chronic pelvic pain. Br J Obstet Gynaecol 1992;99:59. 
24. Maher CF, Carey MP, Dwyer PL, et al. Percutaneous sacral nerve root neuromodulation for intractable interstitial cystitis. J Urol 2001;165:884-886.

25. Goldenberg DL, Burckhardt C, Crofford L. Management of fibromyalgia syndrome. JAMA 2004;292:2388.

26. Affaitati G, Fabrizio A, Savini A, et al. A randomized, controlled study comparing a lidocaine patch, a placebo patch, and anesthetic injection for treatment of trigger points in patients with myofascial pain syndrome: evaluation of pain and somatic pain thresholds. Clin Ther 2009;31:705.

27. Allen LA, Woolfolk RL, Escobar JI, Gara MA, Hamer RM. Cognitive-behavioral therapy for somatization disorder: a randomized controlled trial. Arch Intern Med 2006;166 (14):1512-1518. DOI:10.1001/archinte.166.14.1512. PMID 16864762 .

28. Cahill SP, Foa EB. A glass half empty or half full. Where we are and directions for future research in the treatment of PTSD. In: Taylor S, ed. Advances in the treatment of post-traumatic stress disorder: cognitive-behavioral perspectives. New York. Springer. 2004.p.267-313.

29. Eisenberg E, McNicol ED, Carr DB. Efficacy and safety of opioid agonists in the treatment of neuropathic pain of nonmalignant origin: systematic review and meta-analysis of randomized controlled trials. JAMA 2005;293:3043.

30. Tay SK, Bromwich N. Outcome of hysterectomy for pelvic pain in premenopausal women. Aust N Z J Obstet Gynaecol 1998; 38:72.

31. Papasakelariou C. Long-term results of laparoscopic uterosacral nerve ablation. Gynaecological Endoscopy 1996;5:177.

32. Possover M, Baekelandt J, Chiantera V. The laparoscopic approach to control intractable pelvic neuralgia: from laparoscopic pelvic neurosurgery to the LION procedure. Clin J Pain 2007;23:821.

\section{ABOUT THE AUTHORS}

\section{Jai Kishan Goel (Corresponding Author)}

Professor, Department of Obstetrics and Gynecology, Shri Ram Murti Smarak Institute of Medical Sciences, Bareilly-Nainital Road, Bareilly Uttar Pradesh, India, Phone: 9457213429, e-mail: drjkgoel309 (a)gmail.com

\section{Siddharth Goel}

Assistant Professor, Department of Orthopedics, Subharti Medical College, Meerut, Uttar Pradesh, India 